\title{
Una evaluación parcial de la política habitacional en el Distrito Federal en el último periodo de gobierno
}

\section{A partial evaluation of the housing policy in Distrito Federal, in the latest government period}

Tamayo, Sergio (Coord.) (2007), Los desafíos del Bando 2. EVALUACIÓN MULTIDIMENSIONAL DE LAS POLÍTICAS HABITACIONALES EN EL D. F., 2000-2006, SEDUVI-INVI-UACM-CAM, MÉXICO, 393 PP., ISBN: 13 978-968-9034-01-8.

En diciembre de 2000, a dos días de asumir el poder, el jefe de gobierno del Distrito Federal (GDF) emitió el Bando 2, el cual prohibía la construcción de conjuntos habitacionales en nueve de las 16 delegaciones políticas del Distrito Federal (DF) y la permitía en las cuatro delegaciones centrales. Este edicto, que reivindicaba que la conducción del desarrollo urbano es una responsabilidad del gobierno, generó fuertes y acalorados debates públicos -por parte de los diversos actores vinculados al desarrollo urbano y la vivienda- que se realizaron en foros públicos, medios de comunicación, espacios académicos, el legislativo local, en las puertas de las oficinas de gobierno e incluso en la Suprema Corte de Justicia de la nación. Justo por ello, este libro, realizado por encargo de la Secretaría de Desarrollo Urbano y Vivienda del GDF, encontrará un gran público.

El Bando 2 fue un instrumento de la política de desarrollo urbano que pretendió revertir el crecimiento desordenado de la ciudad, la expansión urbana y el despoblamiento de las áreas centrales; preservar el suelo con valor ecológico; y facilitar el acceso de vivienda a la población pobre en las delegaciones centrales. Sin embargo, este libro especifica sus alcances en el subtítulo: la evaluación de la política habitacional en el sexenio anterior. Así, el libro tiene un nombre más amplio de lo que analiza y la evaluación de otros aspectos que constituyeron parte de los desafíos urbanos y ambientales del Bando están pendientes. ¿Se revirtió el crecimiento desordenado y la expansión de la mancha urbana? ¿Se preservó el suelo con valor ecológico en el DF? 
La evaluación de la política habitacional se pretende abordar de la forma más integral posible. El equipo interdisciplinario que realizó esta evaluación combina investigadores consolidados con otros nuevos, tal vez por ello la calidad de los artículos es desigual. Sin embargo, la mayor parte de ellos está realizada con rigor académico.

\section{La política del Bando 2 y el debate político}

En su artículo, Sergio Tamayo recoge las controversias, el contexto político y la correlación de fuerzas en que se emitió e inició la vigencia del Bando. En el apartado "La pugna por la ciudad", el autor nos recuerda que en el 2000 el Partido de la Revolución Democrática (PRD), en el poder, no tenía mayoría en el legislativo local y que seis delegaciones del DF eran gobernadas por el Partido Acción Nacional (PAN), tres de ellas junto con la Cuauhtémoc constituyen la llamada Ciudad Central. La emisión del Bando provocó reacciones encontradas e impactó de forma diferencial los intereses de los distintos actores, por lo que el debate se anclaba en diversos argumentos. Los empresarios centraron sus críticas en las restricciones a la inversión privada; los legisladores en el autoritarismo de la medida y en el desprecio por el marco jurídico vigente; los académicos en el pragmatismo del decreto; y el Movimiento Urbano Popular (MUP) tuvo distintas posiciones dependiendo de sus intereses específicos y su presencia en el territorio: quienes tenían suelo y proyectos periféricos estaban contra el Bando, y quienes tenían presencia en las delegaciones centrales se favorecieron con esa medida.

Finalmente, en el apartado titulado "Derivaciones", Tamayo expresa que el Bando 2 fue un instrumento que tuvo objetivos nobles en términos urbanos, ecológicos y sociales; y que la vivienda entendida como un derecho humano, fue decididamente apoyada con recursos fiscales.

Una de las críticas que hago de esta contribución es que el autor usa el concepto producción social de la vivienda para referirse a la producción de vivienda de interés social. La primera la han definido claramente las organizaciones no gubernamentales (ONG) como la vivienda que se realiza bajo el control de la población organizada y otros agentes sociales que operan sin fines lucrativos. En tanto que la segunda, la legislación local y federal la definen en función de su costo en veces el salario mínimo. 


\section{El Bando 2 y el marco normativo}

En este artículo, Ramón Tamayo et al. analizan un amplio panorama de la legislación urbana y habitacional en los ámbitos federal y local, para ubicar el Bando en su dimensión normativa y validez jurídica. El análisis concluye, citando una disertación de la Suprema Corte de Justicia de la nación, que el Bando se debería llamar Acuerdo administrativo y que se trata de un instrumento donde convergen lo jurídico y lo programático. Se trata de un acto, concluyen los autores, que legítimamente corresponde al ejecutivo de una novel administración con base en un diagnóstico, en el marco jurídico y en las aspiraciones sociales y políticas de quien asume el poder.

\section{Los programas de vivienda}

Claudia Puebla describe los programas del Instituto de Vivienda del Distrito Federal (Invi) y de forma perspicaz introduce una serie de comentarios e hipótesis acertadas. En primer lugar se explican algunas especificidades del DF, donde hay una gran presencia de organizaciones sociales demandantes de vivienda; no hay suelo urbano apto para la expansión urbana; el gobierno local aplica una política habitacional dirigida a la población excluida del mercado formal; y las ong han influido en el diseño de las políticas públicas. También se apunta la transformación de los organismos federales de vivienda y su impacto en los derechohabientes del DF.

La autora destaca varios aspectos: 1) se aplicó una enérgica política habitacional que hasta 2005 había realizado 130 mil acciones de vivienda (77\% de mejoramiento y $23 \%$ de obra nueva). Se trata -dice- de un nivel de producción de vivienda para población de bajos ingresos sin precedentes en la ciudad y el país. ${ }^{1}$ 2) La política de vivienda se vinculó a la de desarrollo urbano y se adecuó a las especificidades del territorio (vivienda nueva en las áreas centrales y mejoramiento en la periferia). 3) Se atendió a (algunas) familias que habitaban en terrenos vulnerables en la periferia o inmuebles en riesgo en las áreas centrales.

El Programa de Vivienda en Conjunto, que se llevó a cabo en las delegaciones centrales y en tres del llamado Primer Contor-

\footnotetext{
${ }^{1}$ Hay que señalar que el programa Renovación Habitacional Popular, realizado después de los sismos de 1985, reconstruyó en el DF 48,800 viviendas en poco más de dos años.
} 
no, permitió que la población permaneciera en las áreas centrales y dio trabajo a empresas constructoras medianas y pequeñas. Por su parte, el Programa de Mejoramiento Habitacional se realizó fundamentalmente en las delegaciones intermedias del Segundo Contorno y constituye un ejercicio inédito en el país, pues actúa en escala masiva en áreas marginadas con una gestión individual, apoya la vivienda progresiva (la vivienda que se construye progresivamente) y emplea a jóvenes arquitectos, quienes además reciben o mejoran una formación social a través del trabajo directo con la gente.

La autora aborda el tema de las prácticas corruptas y clientelistas que realizan algunos gestores profesionales, pero señala que el Invi no privilegió el otorgamiento de créditos a una organización en particular (el libro incluye un listado de las organizaciones beneficiadas) y modificó sus Reglas de Operación para combatir la cultura de la gestión clientelar.

También se señalan algunas debilidades de los programas de vivienda: el alto nivel de subsidios, la escasa recuperación crediticia, la aplicación de los recursos fiscales dependen de la voluntad del gobierno en turno. ${ }^{2}$ En cuanto al Programa de Mejoramiento: la subcontratación de técnicos; el difícil control de calidad por la dimensión y dispersión del programa, así como la indefinición de la copropiedad familiar. Finalmente, la autora apunta la necesidad de ampliar el programa de mejoramiento habitacional hacia mayores beneficios a los barrios y concluye que atender las necesidades de vivienda de los más pobres requiere la intervención y financiamiento directos del gobierno, sólo así es posible hacer accesibles los créditos a los sectores de menores ingresos.

\section{El proyecto arquitectónico y urbanístico}

Vicente Guzmán evalúa tres proyectos con 64 viviendas realizados por el Invi en la administración anterior: la rehabilitación de un inmueble con valor artístico, una construcción nueva y tres acciones de mejoramiento habitacional. Se trata de un pequeñísimo botón de muestra comparado con las miles de acciones habitacionales realizadas por ese gobierno. De acuerdo con el autor, en la evaluación se tomó en cuenta a la gente y su percepción acerca de la vivienda y el entorno (más bien aparecen algunos relatos sobre la situación anterior a la vivienda nueva, rehabilita-

${ }^{2}$ La voluntad política se mantiene y se ha incrementado, el actual jefe de gobierno del DF se trazó la meta de realizar 200 mil acciones de vivienda en el periodo 2006-2012. 
da o mejorada). A partir de la lectura del texto y del material gráfico, así como de experiencias propias, quedan algunas dudas sobre cómo la gente habita y se apropia de su vivienda. Por ejemplo, analizando los planos de Bucareli (habitado por indígenas mazahuas) y Guanajuato (habitado por otomíes), uno se pregunta por qué no se usan muebles de sala. ¿Los indígenas son pobres o habitan de otra forma el espacio? Tampoco se sabe de qué dimensión son las viviendas, ni la relación de habitantes por $\mathrm{m}^{2}$ construidos o cuartos. Hay algunos proyectos del Invi (tal vez no es el caso de los proyectos analizados) que con seguridad han mejorado las condiciones higiénicas de las viviendas, pero no han resuelto el problema de hacinamiento, pues las viviendas son de dimensiones muy pequeñas.

El autor reitera en los tres casos que analiza, que los proyectos del Invi presentan deficiencias con respecto a dispositivos de ahorro de agua y luz, el reciclaje de aguas usadas, el aprovechamiento del agua de lluvia y la ausencia de un baño - WC-, lavamanos de uso múltiple y simultáneo. Por último, vale destacar que el autor señala que además de vivienda se debería hacer arquitectura y hacer ciudad, es decir, tener en cuenta criterios estéticos en los proyectos habitacionales y favorecer las relaciones con otra gente y con su entorno social y urbano.

El artículo no menciona el rico proceso de acompañamiento (que incluyó un ejercicio de diseño participativo) del proyecto de los otomíes que desde 1995 realizó la oNG Casa y Ciudad, ni que este proyecto ganó el Premio Nacional de Vivienda otorgado por el gobierno federal en 2004.

\section{La participación ciudadana en los programas de vivienda}

Javier de la Rosa y Luis Luna analizan las diversas concepciones sobre la política pública que se generan en el marco de las reformas económicas recientes y buscan la cooperación de las organizaciones sociales y civiles sin fines de lucro, para resolver necesidades sociales con mecanismos de mercado. Esta visión -dicencontrasta con la del GDF, quien reconocía la función social del gobierno y su responsabilidad para atender los rezagos sociales. Los autores destacan que la política social territorializó y priorizó las zonas marginadas.

En el apartado "La perspectiva de los actores" se discute la relación de las ONG con el gobierno. Aquí un funcionario señala que el Invi realizaba el programa de mejoramiento habitacional 
que pensaron las ONG y que por eso cooptaron a algunos de sus miembros y a otros del MUP (en este proceso un ícono de la lucha urbana, Superbarrio, también cambió de ring). Además se argumenta que por la magnitud del programa era imposible la participación de las ONG, pero que a estas instituciones se les contrató para realizar asesoría técnica. Así, por un lado se reconoce que el programa que se impulsa es el que diseñaron las ONG, pero por otro, se les asigna el mismo papel que a cualquier joven arquitecto independiente.

Los autores señalan que el Programa de Mejoramiento de Vivienda considera al ciudadano en función del acceso individual al programa, de ahí que digan que en este caso la ciudadanización equivale a individualización. En este sentido se incluyen comentarios de un funcionario y un representante del MUP, quienes aseguran que ese programa no fortalece la organización ni el tejido social. En contraparte, otro funcionario del Invi señala que al Mup no le interesaba ese programa porque no puede controlar la clientela.

En este artículo no se aborda el tema de la participación social en los programas habitacionales de las áreas centrales (Vivienda en Conjunto). Además, es curioso que el libro incluya una imagen de una Unión Popular Democrática que textualmente dice “¿No tienes vivienda propia?” y después señala que "la vivienda es un derecho humano no una mercancía”, p. 238 (tal vez no hay contradicción, el derecho a la vivienda se entiende como el derecho a la propiedad de una vivienda).

\section{La actuación de los desarrolladores habitacionales privados}

El artículo de Teresa Esquivel explora las transformaciones recientes de la Ciudad Central entre 2000 y 2005, a partir del análisis de datos del Instituto Nacional de Estadística, Geografía e Informática (INEGI) y de la acción habitacional privada, a través de dos fuentes: 1) los créditos ejercidos por distintos organismos financieros en todo el DF (en número de viviendas) y 2) la oferta habitacional real en cuatro delegaciones centrales (en número de proyectos inmobiliarios). Esquivel evidencia que en el 2000 el proceso de despoblamiento abarcaba ocho delegaciones (y no sólo a cuatro), pero que merced a la política de redensificación del GDF este proceso se revirtió en dos delegaciones (Cuauhtémoc y Miguel Hidalgo), la tasa de despoblamiento se aminoró en otras dos y la pérdida de vivienda se revirtió en las cuatro delegaciones centrales. 
De 150 mil créditos de vivienda nueva ejercidos en el periodo 2001-2005 (incluye 72,912 créditos otorgados por el Invi, aunque este no un organismo privado): la mayor parte (60\%) se ejerció en el Primer Contorno ${ }^{3}$ y en la Ciudad Central se ejerció casi la tercera parte.

Aquí claramente se reconoce que la emisión del Bando 2 implicó el incremento en el costo del suelo y, por ello, la oferta privada se orientó mayoritariamente a la vivienda media y media alta, es decir, a la población con ingresos mayores. La autora analiza el tipo de oferta habitacional en los distintos territorios de la Ciudad Central, lo que sigue las pautas históricas del poblamiento; esto es, no se alcanzan a ver efectos de aburguesamiento o gentrificación: la vivienda de interés social se construye en colonias proletarias, mientras que la vivienda media y media alta se construye en antiguas colonias aristocráticas y colonias modernas.

Con datos del INEGI, la autora analiza los cambios en los tipos de vivienda de las cuatro delegaciones centrales: los departamentos se incrementaron en 43 mil unidades, las casas independientes decrecieron en 22 mil unidades, las vecindades decrecieron en $11 \%$ y 7 mil cuartos y cuartos de azotea también disminuyeron en casi dos mil unidades. Así, las viejas casonas deterioradas o cuyos dueños no cuentan con recursos para mantenerlas, se constituyeron en la reserva de suelo para edificar los nuevos departamentos.

Finalmente, Esquivel advierte que el incremento en el costo del suelo puede contribuir al desplazamiento de la población de bajos ingresos de las áreas centrales y a la ocupación de zonas verdes con valor ecológico, lo que pone en entredicho los propósitos del Bando 2.

\section{Política de gestión y dinámica de suelo}

El autor intenta (sin conseguirlo) analizar las políticas y estrategias de acceso al suelo por parte de los sectores público y privado. Este artículo difunde información pública sobre predios gestionados por las organizaciones sociales y los montos de crédito autorizados por el Invi, así como precios del suelo extraídos de un $M a-$

\footnotetext{
${ }^{3}$ Aquí se incluye a Iztapalapa, Azcapotzalco y Gustavo A. Madero (las dos últimas delegaciones también pierden población). Vale recordar que el Bando 2 prohibía expresamente la construcción de conjuntos de vivienda mayores a 35 unidades en nueve delegaciones (entre ellas Iztapalapa) y que no mencionaba a Azcapotzalco, Gustavo A. Madero e Iztacalco, donde el sector público y el privado construyeron una gran cantidad de viviendas.
} 
nual de Procedimientos y Lineamientos Técnicos de Evaluación Inmobiliaria que el GDF publica anualmente en la Gaceta Oficial. Sin embargo, en el caso de la información del Invi, el autor mismo reconoce que los costos promedio de las acciones por vivienda no están desglosados. Así, no se sabe si el costo corresponde a las acciones de construcción nueva o de mejoramiento, o si ambos están promediados, y tampoco se sabe cuál es el monto correspondiente a la adquisición del suelo, tema del artículo.

Además, la información (proveniente de anuncios en la prensa) que se presenta sobre el mercado de suelo y vivienda no se desglosa ni se territorializa, por lo que prácticamente no dice nada. El mercado inmobiliario es muy complejo y se dirige a distintas clientelas; entonces poco dicen los valores promedio de suelo y vivienda por año para todo el DF o la Ciudad Central. Esa información se debería desglosar de acuerdo con la oferta: terrenos, tipo de departamentos o casas (dimensión, estado físico, número de baños y estacionamientos), construcciones nuevas o usadas, etcétera. Además de considerar la ubicación y normatividad urbana aplicable, pues un terreno o departamento de dimensiones iguales no vale lo mismo a veces en una misma calle.

En este artículo no se menciona nada sobre 1) la desincorporación del suelo propiedad del GDF en favor de proyectos habitacionales, 2) la compra de inmuebles y baldíos para la construcción de vivienda en las delegaciones centrales, lo que constituía parte de la política de suelo del Invi y 3) información sobre las decenas de predios expropiados por el GDF. ¿En cuántos casos se presentaron los dueños a reclamar su propiedad o indemnización? ¿El costo del suelo fue cubierto o no por el crédito del Invi y en qué casos y cuánto se subsidió?

\section{Políticas de redensificación y su impacto en la estructura urbana}

Salomón González alude a un movimiento generalizado (en la literatura anglosajona) de retorno al modelo de la ciudad compacta que abarca varios temas: el desarrollo sustentable, el despoblamiento de las áreas centrales, la presión sobre las áreas verdes periféricas; la accesibilidad y el incremento del parque vehicular particular. Vale señalar que esta discusión también es un tema en el debate latinoamericano sobre las políticas urbanas y la ciudad del futuro, y que algunas ciudades como Santiago de Chile, Sao Paulo, Quito y la ciudad de México han desarrollado 
políticas urbanas que pretenden aprovechar la ciudad construida y evitar la expansión periférica.

El autor tiene un fresco estilo provocador que pone en tela de juicio cosas que se dan como verdades irrebatibles. Él pregunta si el debate de fondo es el del repoblamiento, las densidades o el de la distribución espacial de los recursos urbanos. Asimismo, cuestiona a los defensores de la ciudad compacta, pues -según él- no hay evidencias contundentes, sino juicios de valor, sobre los beneficios y/o efectos negativos de la expansión urbana o la ciudad dispersa. A esta discusión teórica había que ponerle nombre, lugar y tiempo, pues puede ser que en los albores del siglo XXI ciudades como Quebec, Barcelona o Puebla aún aguanten una mayor dispersión o urbanización periférica. Pero cabe preguntarse si la ciudad de México y su Zona Metropolitana resisten más desarrollos habitacionales periféricos de alta y baja densidad como los que se realizaron recientemente. Más allá de esta discusión se tiene presente que el modelo de urbanización periférica en escala metropolitana continúa siendo atractivo para distintos actores (las grandes constructoras, algunas autoridades locales y federales y población de distinto estrato socioeconómico), porque allí el suelo sin servicios es más barato.

El autor reconoce que la ciudad de México continúa creciendo a ritmos menores que antes y que su estructura territorial ha transitado de una ciudad con centralidad hegemónica a una policéntrica. Sin embargo, los instrumentos de planeación y gestión metropolitana están rezagados y aún no se adaptan a la nueva realidad. Finalmente, el autor demuestra lo que otros investigadores conocen o sospechan: la Ciudad Central concentra población inmigrante y población adulta, y es una zona que expulsa a familias jóvenes con hijos a la periferia. De acuerdo con su análisis, la producción de vivienda pública es la variable que mejor explica la redistribución de la población en la Zona Metropolitana de la Ciudad de México. Conviene señalar que los datos estadísticos del INEGI que utilizó el autor, no coinciden con los difundidos oficialmente por esa institución, por ello sus resultados indican que la tasa de despoblamiento, aunque aminorada, continuaba en 2005 en toda la Ciudad Central. Sin embargo, en este mismo libro, Teresa Esquivel (p. 274) demuestra que en dos delegaciones el proceso de despoblamiento se revirtió. 


\section{Temas ausentes en el libro}

No se aborda un aspecto fundamental en la evaluación de las políticas habitacionales: la recuperación de los créditos. Varios autores del libro concuerdan en que la vivienda es un derecho y que el GDF contribuyó a su cumplimiento. Sin embargo, el otorgamiento de créditos públicos blandos para el acceso a la propiedad privada de un alojamiento implica ciertas obligaciones que la población debe cumplir para garantizar el retorno de recursos y, con ello, que otras familias puedan acceder a nuevos créditos. Salvo alguna mención en el artículo de Claudia Puebla, nada se dice acerca de la recuperación crediticia y de la cultura del no pago.

Tampoco se trata el tema de la normatividad urbana. De acuerdo con diversas evidencias empíricas, el sector privado negocia mejor la normatividad urbana y construye mayor densidad edilicia que el sector público.

Un efecto no estudiado es el del mercado de vivienda usada, que también se disparó con el Bando 2, tanto por el costo de los nuevos departamentos como por la dimensión de las viviendas nuevas. Incluso varias de las acciones de vivienda que realizó el Invi en el periodo estudiado consistieron en la adquisición de vivienda usada.

No se explora el impacto del Programa de Mejoramiento de Vivienda en la economía del barrio. En este programa, varias familias adquirirían materiales de construcción en distribuidoras de barrio y contratarían mano de obra local (plomeros, electricistas, albañiles, etcétera).

Tampoco se profundiza en la investigación de los programas emergentes, programas nobles pero con resultados insuficientes para enfrentar la situación de riesgo en que habitan centenas de familias. Generalmente las acciones emergentes, por no planificarse, encarecen los costos de operación de los programas, pues deben reubicar a decenas de familias en vivienda transitoria y erogan cuantiosos recursos en ayudas para renta.

\section{Comentarios finales}

En el libro aparece en dos ocasiones un argumento que pretende presentar el incremento del costo del suelo en las delegaciones centrales, motivado por el Bando 2, como un efecto planificado y una acción pública intencionada. En la página 280, y sobre todo en la 377 , se dice que una manera de rehabilitar el centro impli- 
caba justamente revalorizarlo, es decir, darle un valor que no tenía. Se trata de un argumento que tal vez pretende defender o justificar la acción de un gobierno bien intencionado, pero que no está expresada de forma directa ni indirecta en el Bando. Por el contrario, este edicto señala expresamente que en las delegaciones centrales se construiría vivienda para la población humilde. ¿Se podría lograr eso incrementando el costo del suelo? ¿Era una intención planificada pagar más por el valor del suelo para desarrollar vivienda para la población humilde?

Por otro lado, la lectura del libro provoca la reflexión en profundidad sobre dos temas cruciales: uno es la continuidad de las políticas públicas en una ciudad y un país, que de acuerdo con los usos y costumbres de los diversos partidos políticos, cada cambio de gobierno (incluso del mismo partido) implica la reinvención de las mismas. El otro es el de la dimensión metropolitana, pues la ciudad real, la que rebasa los límites jurídico-políticos, requiere urgentemente de políticas metropolitanas: mientras en el DF se efectuaron restricciones a la construcción de conjuntos habitacionales en sus áreas periféricas, en varios municipios de la periferia metropolitana se construyeron miles de viviendas casi todas iguales y de dimensiones cada vez más infames, que expanden la ciudad real a territorios cada vez más distantes. ¿̇Se puede y se debe seguir diseñando y aplicando las políticas públicas de manera local sin tener en cuenta el territorio de la ciudad real, más allá de las atribuciones que a cada gobierno local otorga el marco jurídico vigente?

Recibido: 9 de enero de 2008. Aceptado: 28 de enero de 2008.

Víctor Manuel Delgadillo-Polanco Universidad Autónoma de la Ciudad de México Victor_delgadill@hotmail.com

Víctor Manuel Delgadillo Polanco. Es doctor en urbanismo por la Universidad Nacional Autónoma de México (UNAM) y maestro en arquitectura y planeación urbana por la Universidad de Stuttgart. Actualmente es profesor de tiempo completo en El Colegio de Humanidades y Ciencias Sociales de la Universidad Autónoma de la Ciudad de México. Consultor de la unEsCo para la elaboración del Programa de Ordenamiento Urbano Ambiental y 
Plan de Gestión para la recuperación del patrimonio cultural y natural de Xochimilco, entre 2005 y 2006. Miembro del Sistema Nacional de Investigadores (SNI), nivel I, desde enero 2007. Obtuvo la Medalla Alfonso Caso por su doctorado en urbanismo, otorgada por el Consejo Universitario y la Comisión al Mérito Universitario de la UNAM, marzo 2007. Sus líneas de investigación actual son: urbanismo y patrimonio cultural en América Latina, actores sociales y políticas públicas, políticas de reciclamiento y desarrollo urbano, vivienda en áreas urbanas centrales. Entre sus publicaciones recientes se encuentran: en coautoría, Hábitat popular en los centros antiguos de Iberoamérica, Programa Iberoamericano de Ciencia y Tecnología para el Desarrollo, Red XIV.F Tecnologías Sociales para la Producción Social del Hábitat, México (2007); "La gran urbe latinoamericana: polarizada, fragmentada, globalizada, ¿e ingobernable?", Seminario Divide e Incluye, nueva gobernabilidad en ciudades fragmentadas de América Latina, 19 y 20 de junio, Fundación Heinrich Böll-Habitat Unit TU Berlín, http://www.boell.de/downloads/latein/teile-beteilige/delgadillo_La-gran-urbe-latinoamericana.pdf) (2007); "De habitantes suicidas y simulacros: 20 años de atención a los tugurios del centro histórico", Ciudades, 74, Red Nacional de Investigación Urbana, México, pp. $40-46$ (2007); “Ordenamiento urbano y asentamientos irregulares", en Ciro Caraballo (coord.), Xochimilco, Tláhuac y Milpa Alta, resumen del plan integral y estructura de gestión del polígono inscrito en la lista del Patrimonio Mundial, unesco, México, pp. 33-34 (2007). 